\title{
Immune activation and arterial stiffness in lean adults with HIV on antiretroviral therapy
}

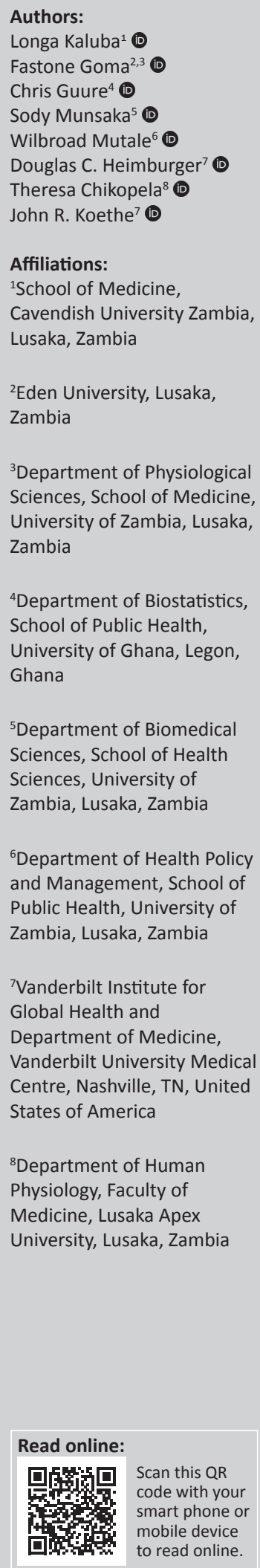

Background: Greater T-cell activation was associated with reduced vascular compliance amongst persons living with HIV (PLWH) especially among overweight and obese individuals. There is a paucity of data regarding immune activation and arterial stiffness amongst PLWH in sub-Saharan Africa (SSA).

Objective: To determine the association between immune activation and arterial stiffness in lean PLWH in SSA.

Method: Forty-eight human immunodeficiency virus positive (HIV+) adults on antiretroviral therapy $\left(\right.$ ART) $>5$ years and 26 HIV-negative adults, all with $\mathrm{BMI}<25 \mathrm{~kg} / \mathrm{m}^{2}$ and no history of CVD, were enrolled. The relationship of vascular compliance with circulating CD4+ and CD8+ naïve, memory, activated and senescent $\mathrm{T}$ cells, and serum 8-isoprostane was assessed by HIV status.

Results: Increased immune activation was observed in the CD4+ and CD8+ T cells of PLWH, $16.7 \%$ vs. $8.9 \%$ and $22.0 \%$ vs. $12.4 \%$ respectively; $p<0.001$ (both). Furthermore, a higher proportion of senescent CD4+ T cells were associated with a lower carotid-femoral pulse wave velocity (cfPWV; $p=0.01$ ), whilst a higher proportion of activated CD8+ T cells were associated with a lower carotid-radial pulse wave velocity (crPWV; $p=0.04)$, after adjustment for BMI and age. However, PLWH also had a higher median carotid-femoral augmentation index (cfAiX) $(21.1 \%$ vs. $6.0 \% ; p<0.05)$ in comparison to their HIV controls.

Conclusion: Our population of lean PLWH had increased immune activation and higher cfAiX, a marker of arterial stiffness, compared to HIV-negative persons. The negative association between immune activation and arterial stiffness as measured by crPWV in PLHW on long-term treatment needs further elucidation.

Keywords: endothelial dysfunction; immune activation; lean adults; antiretroviral therapy; arterial stiffness.

\section{Introduction}

The prevalence of non-communicable diseases in sub-Saharan Africa (SSA) has continued to rise over the last two decades ${ }^{1,2}$ with cardiovascular-related disorders being a leading cause of death in persons living with HIV (PLWH). ${ }^{3}$ Endothelial dysfunction, a proposed mechanism leading to this increased cardiovascular disease (CVD) risk, results in diminished relaxation of the vascular smooth muscle and to arterial stiffness. ${ }^{4,5,6}$

In the general population, a meta-analysis ${ }^{7}$ found that an increase of 1 metre per second $(\mathrm{m} / \mathrm{s})$ in pulse wave velocity (PWV), a marker of greater arterial stiffness, was associated with a $14 \%$ increased risk of cardiovascular events and 15\% increased risk of all-cause mortality when adjusted for age, sex and cardiovascular risk factors. Increased arterial stiffness and immune activation have been reported in PLWH. 8,9

Persons living with human immunodeficiency virus (HIV) have reduced levels of endothelial nitric oxide synthase (eNOS), an important regulator of vascular compliance. ${ }^{10}$ Further, other factors such as immune activation, inflammation, oxidative stress and HIV proteins have also been associated with impaired vascular compliance in PLWH.

Corresponding author: Longa Kaluba, kalubalonga@gmail.com

Dates: Received: 04 Nov. 2020 | Accepted: 02 Dec. 2020 | Published: 19 Mar. 2021

How to cite this article: Kaluba L, Goma F, Guure C, et al. Immune activation and arterial stiffness in lean adults with HIV on antiretroviral therapy. S Afr J HIV Med. 2021;22(1), a1190. https://doi.org/10.4102/sajhivmed.v22i1.1190

Copyright: ㄷ 2021. The Authors. Licensee: AOSIS. This work is licensed under the Creative Commons Attribution License. 
Immune activation is increased in PLWH compared to HIVnegative persons. ${ }^{6,11}$ Recent studies, predominantly from cohorts in the United States (US), report associations between circulating T-cell subsets and vascular compliance in PLWH. Kaplan, Sinclair ${ }^{12}$ reported a positive association between activated CD4+ T cells and arterial stiffness after adjusting for HIV ribonucleic acid (RNA) and total peripheral CD4+ T-cell count. A longitudinal study by Karim, Mack ${ }^{9}$ also reported a positive correlation between activated CD4+ $\mathrm{T}$ cells and carotid arterial stiffness both pre- and post-highly active antiretroviral therapy (HAART) initiation. CD8+ T-cell activation was associated with reduced smooth muscle relaxation independent of other cardiovascular risk factors in PLWH with viral suppression..$^{13,14}$

Whilst the mechanistic link between T-cell activation and vascular compliance is not fully understood, immune cells are capable of generating reactive oxygen species and contributing to oxidative stress. ${ }^{15}$ Higher levels of reactive oxygen species and circulating pro-inflammatory cytokines, two conditions commonly increased in PLWH despite effective plasma viral suppression on antiretroviral therapy (ART), contribute to endothelial dysfunction and reduced vascular compliance. ${ }^{16,17,18,19,20}$ Higher 8-isoprostane, a marker of oxidative stress, is associated with subsequent all-cause mortality in PLWH independent of CD4+ T-cell count and high-sensitivity C-reactive protein (hsCRP). ${ }^{21}$ The overproduction of reactive oxygen species such as superoxide, which is formed when oxygen is reduced by a single electron, has been associated with the progression of cardiovascular disorders. ${ }^{18}$ Increased superoxide binds to the increased nitric oxide (NO) to form peroxynitrite, which results in the production of a powerful oxidant that traverses into the endothelial cell and directly damages the deoxyribonucleic acid (DNA); compromising the bioavailability of $\mathrm{NO}$ and resulting in reduced relaxation. ${ }^{16,18}$ In addition, HIV proteins have also been implicated in endothelial dysfunction. ${ }^{22}$

In SSA, the burden of CVD in PLWH has tripled over the last two decades. ${ }^{23}$ However, much of our current understanding of HIV and cardiovascular function arises from cohort studies in the US and Europe, which often include a high proportion of overweight and obese individuals reflective of the general population. ${ }^{24}$ Whilst rates of obesity are rising in many SSA countries, particularly South Africa, there remains a broad distribution of body composition amongst PLWH in the region which includes a substantial number of individuals with lower body mass index (BMI) values. At present, there is a paucity of data on whether inflammation and immune activation in 'lean' (i.e. BMI $<25$ kilogram per square metre $\left.\left[\mathrm{kg} / \mathrm{m}^{2}\right]\right)$ PLWH is accompanied by a similar degree of arterial stiffness as observed in higher BMI individuals. To this end, we assessed relationships between cellular immune activation, oxidative stress and arterial stiffness in a cohort of lean PLWH on long-term ART.

\section{Materials and methods}

We conducted an analytical cross-sectional study at University Teaching Hospital (UTH) in Lusaka, Zambia between September 2018 and June 2019 amongst PLWH on long-term ART with BMI's $<25 \mathrm{~kg} / \mathrm{m}^{2}$. Persons accompanying patients to the ART clinic and general medicine outpatients department were recruited as HIV-negative controls. The HIV infection status was confirmed in all HIV-negative participants by rapid test. All PLWH were on a regimen of efavirenz, emtricitabine, and tenofovir disoproxil fumarate for more than 5 years. Persons who were pregnant, or with known CVD, rheumatologic disease or any other self-reported pathology, active infectious conditions aside from HIV, or with a history of diabetes and habit of tobacco smoking were excluded. Socio-demographic data were collected using the WHO STEPS questionnaire. ${ }^{25}$ Randomly sampled participants provided written informed consent, and ethical approval was obtained.

\section{Endothelial dysfunction measurements}

Carotid-femoral pulse wave velocity (cfPWV) and carotid-radial pulse wave velocity (crPWV) are measures of arterial stiffness, calculated from the time taken for the arterial pulse to propagate from the carotid to the femoral or radial artery, respectively. Carotid-femoral pulse wave velocity is said to be the gold standard in the measure of aortic stiffness. ${ }^{26}$ Other derivatives of PWV measurement include augmentation index (AiX) which is the measure of the enhancement of the central aortic artery reflective wave and is said to be a more sensitive marker for vascular compliance. ${ }^{27}$ All these parameters were measured using the ALAM Complior Analyse device (ALAM medical, France). Non-invasive probes were applied to the surface of the skin over the carotid, femoral and radial arteries with participants lying in a supine position, after resting for a minimum of 5 min. Participants were not allowed to move, speak, or sleep during the measurements. ${ }^{28}$

\section{Biochemical measurements}

Fasting blood was collected in an ethylenediamine tetraacetic acid (EDTA) tube and analysed by flow cytometry. A lyse-wash protocol (Becton Dickinson) was used to stain lymphocytes. A 100 microlitres $(\mu \mathrm{L})$ of whole blood was stained with the following monoclonal antibodies: CD3 APC (Sigma Aldrich, clone MEM-57), CD4 PerCP (Thermofisher, clone RM4-5), CD8 APC-Cy7 (Biolegend, clone HIT8a), HLA DR PE-Cy7 (Thermofisher, clone L243), CD57 PE (Thermofisher, CD57, clone TB01), CD45RA FITC (Sigma Aldrich, clone MEM-56). Fluorescence-activated cell sorting (FACS) lysing solution was added, followed by centrifugation to isolate the WBCs. ${ }^{29}$

T-cell subsets were identified using sequential gating on a FACSverse flow cytometer using FACSuite software (Becton 
Dickinson, San Diego, CA). Lymphocytes were gated using forward, and side scatter (forward scatter [FSC] and side scatter [SSC]) (Appendix Figure 1).

The cells were then gated by the expression of fluorochromes. To overcome the physical overlap of the emission spectra of common fluorochromes, unstained and single stained controls, and fluorescence minus one (FMO) controls were used as a reference to automatically generate compensation matrices using the Flowjo version 10 software. These matrices were manually verified.

\section{Oxidative stress measurements}

Serum samples stored at $-80^{\circ} \mathrm{C}$ were thawed for measurement of 8-isoprostane. An enzyme-linked immunosorbent assay (ELISA) was performed using an immobilised monoclonal antibody of 8-isoprostane (Detroit R\&D, Cat \# 8iso1) as specified by the manufacturer's instructions. ${ }^{30}$

\section{Statistical analyses}

Because of inconsistent distribution of outcome variables, medians and interquartile ranges were calculated for continuous variables and percentages for categorical variables. The relationships between clinical and demographic characteristics were assessed using Mann-Whitney U test or chi-squared test as appropriate. We assessed whether T-cell subsets were associated with markers of endothelial function (crPWV, cfPWV, carotid-radial augmentation index [crAiX], carotid-femoral augmentation index [cfAiX]) and oxidative stress (8-isoprostane) using multivariate models adjusted for age and BMI in the PLWH. The normality of the outcome data was assessed using Q-Q plots and validated with the ShapiroWilk test. Only crPWV required log-transformation.

Multiple linear regression models were used to assess relationships between the independent variables (CD4+ and CD8+ T-cell subsets) and the dependent variables (PWV, AiX and 8-isoprostane); these models were adjusted for BMI and age in the HIV+ group only, because of the small sample size.

Linear regression models incorporating an interaction between CD4+ and CD8+ T-cell subsets with HIV status, adjusted for BMI and age, were also performed (Appendix Table 1-A1). The results of regression models are reported using Beta $(\beta)$ coefficients, confidence intervals $(\mathrm{CI})$ and $p$-values. Analyses were performed using STATA version 15 software.

\section{Ethical considerations}

Participants provided written informed consent, and approval was obtained from the University of Zambia Biomedical Research Ethics Committee (UNZABREC reference number 003-01-18) and the National Health Research Authority (NHRA).

\section{Results}

A total of 74 adult participants were enrolled in the study: 48 with HIV (28 female; 20 male) and 26 HIV-negative (15 female; 11 male). The median age of the PLWH was 41 years versus 23 years amongst the HIV-negative group (Table 1). People living with HIV had a lower BMI compared to the HIV-negative group $\left(18.9 \mathrm{~kg} / \mathrm{m}^{2}\right.$ vs. $20.7 \mathrm{~kg} / \mathrm{m}^{2}$, respectively) but had comparable waist circumferences (72 centimetres $[\mathrm{cm}]$ vs. $70 \mathrm{~cm})$. Median central blood pressure measurements were also comparable between groups (112/78 vs. 116/77). Both cfPWV and crPWV fell within normal ranges of $9.1 \mathrm{~m} / \mathrm{s} \pm 3.2 \mathrm{~m} / \mathrm{s}^{31,32}$ (Table 2). Carotid-femoral augmentation index was significantly higher in PLWH (Table 2). Other measurements of endothelial function and 8-isoprostane were similar between groups.

Naïve CD8+ cells were lower in PLWH compared to the HIV-negative controls $(p<0.001)$. Both activated and memory $\mathrm{CD} 4+$ and CD8+ $\mathrm{T}$ cells were significantly higher in the PLWH as well (Table 3). Higher activated CD8+ T cells were associated with lower crPWV only in the PLWH $(p=0.04)$. Activated CD4+ T cells were significantly associated with 8-isoprostane (Table 4). A 1\% increase in activated CD4+ T cells correlated with a 0.11 picogram $/$ microliter $(\mathrm{pg} / \mu \mathrm{L})$ decrease in 8-isoprostane. Higher senescent CD4+ T cells were associated with lower cfPWV (Table 4). A 1\% increase in senescent CD4+ $\mathrm{T}$ cells corresponded to a $0.22 \mathrm{~m} / \mathrm{s}$ decrease in the cfPWV. These relationships were absent or differed in HIV-negative persons (see Appendix Table 2-A2); notably, greater senescent CD4+ T cells was associated with higher cfPWV in this group (a finding contrary to that of participants with HIV).

Higher memory CD4+ T cells were associated with lower 8 -isoprostane concentrations $(p<0.001)$. Each per cent increase in memory CD4+ T cells correlated with a $0.09 \mathrm{pg} / \mu \mathrm{L}$ decrease in 8-isoprostane. Higher memory CD8+ cells were associated with higher crPWV (Table $4 ; p=0.01$ ). Higher naïve CD8+ cells were associated with higher logtransformed, crPWV (Table 4; $p=0.01$ ).

Finally, we performed an interaction analysis given the differing directionality of the relationship between senescent CD4+ $\mathrm{T}$ cells and cfPWV in the participants with, versus without, HIV. After adjusting for age and BMI, the relationship of senescent CD4+ T cells with cfPWV differed by HIV status (Figure 1 and Appendix Table 3-A3; interaction term $\beta=-0.22 ; p=0.05)$. Similarly, the relationship of senescent CD4+ $\mathrm{T}$ cells with carotid-radial augmentation index differed by the HIV status (Figure 2 and Appendix Table 4-A4; interaction term $\beta=-3.46 ; p=0.03$ ).

\section{Discussion}

In the cohort of lean individuals in Lusaka, Zambia, PLWH on long-term ART had greater arterial stiffness (through increased (fAiX), immune activation, and more senescent 
TABLE 1: Clinical and demographic characteristics.

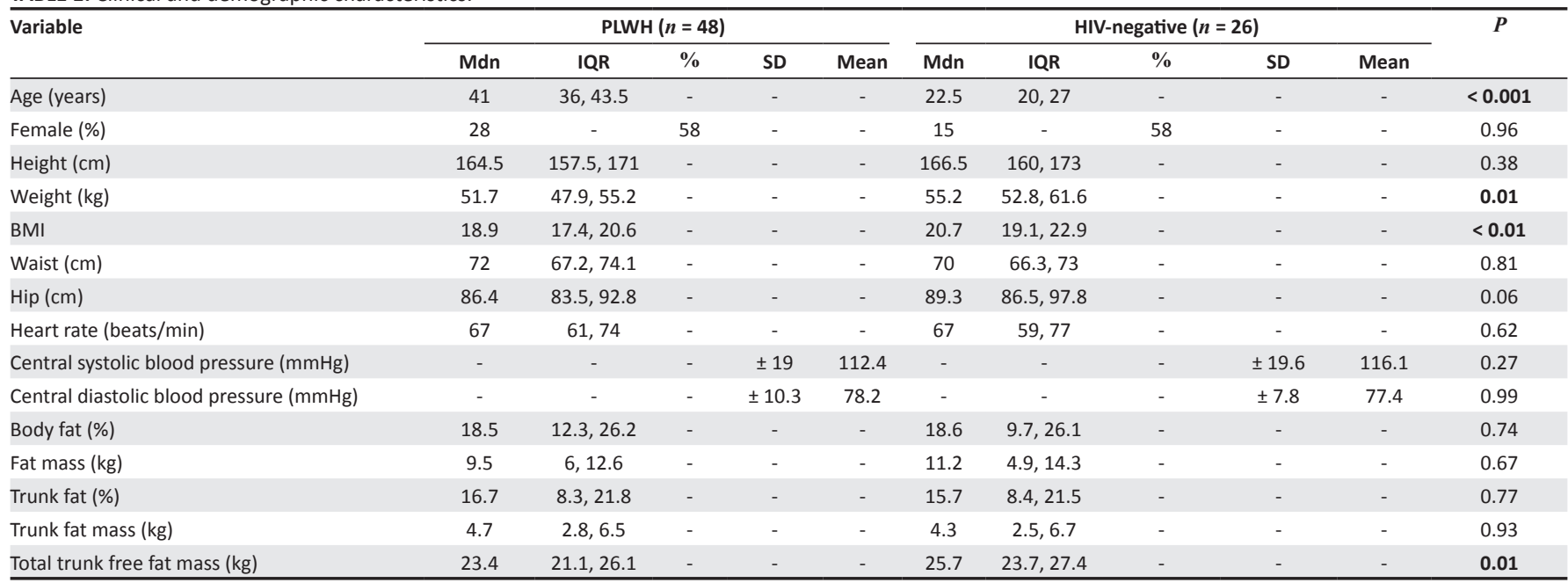

Note: Medians and interquartile ranges. Body composition measurements utilised body impedance analysis (BIA) by the Tanita BC418 MA. Mann-Whitney U test or chi-squared test was used. $p$-values $<0.05$ are shown in bold.

HIV, human immunodeficiency virus; PLWH, persons living with HIV; BMI, body mass index.

TABLE 2: Pulse wave velocity and oxidative stress indices.

\begin{tabular}{|c|c|c|c|c|c|}
\hline \multirow[t]{2}{*}{ Variable } & \multicolumn{2}{|c|}{ PLWH $(n=48)$} & \multicolumn{2}{|c|}{ HIV-negative $(n=26)$} & \multirow[t]{2}{*}{$P$} \\
\hline & Median & IQR & Median & IQR & \\
\hline \multicolumn{6}{|c|}{ Pulse wave velocity indices } \\
\hline $\operatorname{cfPWV}(\mathrm{m} / \mathrm{s})$ & 7.3 & $6.1,8.8$ & 7.0 & $5.8,7.7$ & 0.15 \\
\hline $\operatorname{crPWV}(\mathrm{m} / \mathrm{s})$ & 9.9 & $8.8,10.8$ & 8.7 & $7.3,10.8$ & 0.09 \\
\hline cfAiX & 21.1 & $5.9,35.6$ & 6.0 & $-5.7,24.5$ & 0.04 \\
\hline crAiX & 12.9 & $2.7,41.4$ & 13.7 & $-9.7,26.3$ & 0.20 \\
\hline \multicolumn{6}{|l|}{ Oxidative stress } \\
\hline 8-isoprostane $(\mathrm{pg} / \mu \mathrm{L})$ & 2.6 & $0.9,3.7$ & 1.1 & $0.6,2.8$ & 0.17 \\
\hline
\end{tabular}

Note: Medians and interquartile ranges. Mann-Whitney $\mathrm{U}$ test or chi-squared test was used. $p$-values $<0.05$ are shown in bold.

HIV, human immunodeficiency virus; cfAiX, carotid-femoral augmentation index; crAiX carotid-radial augmentation index; PLWH, persons living with HIV; cfPWV, carotid-femora pulse wave velocity; crPWV, carotid radial pulse wave velocity.

TABLE 3: T-cell subsets.

\begin{tabular}{|c|c|c|c|c|c|}
\hline \multirow[t]{2}{*}{ Variable } & \multicolumn{2}{|c|}{ PLWH $(n=48)$} & \multicolumn{2}{|c|}{ HIV-negative $(n=26)$} & \multirow[t]{2}{*}{$P$} \\
\hline & Median & IQR & Median & IQR & \\
\hline \multicolumn{6}{|l|}{ CD4 T-cells (\%) } \\
\hline CD45RA+ (naïve) & 20.0 & $11.1,28.1$ & 25.3 & $17.7,33.2$ & 0.06 \\
\hline CD45RA- (memory) & 78 & $69,87.6$ & 72.4 & $64.3,77.9$ & 0.04 \\
\hline HLADR-CD57+ (senescent) & 4.4 & $2.7,6.5$ & 3.2 & $2.0,5.6$ & 0.28 \\
\hline HLADR+CD57- (activated) & 15.9 & $9.2,21.7$ & 8.4 & $6.3,10.9$ & $<0.001$ \\
\hline \multicolumn{6}{|l|}{ CD8 T-cells (\%) } \\
\hline CD45RA+ (naïve) & 42.6 & $31.8,52.2$ & 55.0 & $49.0,65.1$ & $<0.001$ \\
\hline CD45RA- (memory) & 52.7 & $44.7,63.7$ & 41.7 & $29.5,46.3$ & $<0.001$ \\
\hline HLADR+CD57- (activated) & 21.55 & $13.2,30.2$ & 12.0 & $6.8,15.2$ & $<0.001$ \\
\hline
\end{tabular}

Note: \% total CD4 and CD8 T cells, respectively; Medians and interquartile ranges. MannWhitney $\mathrm{U}$ test was used. $p$-values $<0.05$ are shown in bold.

HIV, human immunodeficiency virus; PLWH, persons living with HIV; CD4, cluster of differentiation $4 ; C D 8$, cluster of differentiation 8 .

T cells compared to HIV-negative persons, which is consistent with studies conducted in other settings. ${ }^{8,9}$ However, amongst the lean PLWH in our study higher proportions of senescent CD4+ $\mathrm{T}$ cells and activated CD8+ T cells were negatively associated with arterial stiffness as measured by PWV ( $\beta=$ -0.22 and -0.01 , respectively). This finding is in contrast to studies in predominantly US cohorts with a high proportion of overweight and obese participants, in which greater T-cell activation (measured by CD38+HLADR+) has been associated with increased arterial stiffness, hypoxia-induced relaxation and increased atherosclerosis. ${ }^{9,12,13,14}$ Furthermore, most of the associations between T-cell subsets and vascular compliance were not observed in the HIV-negative controls. Whilst this may have been because of fewer participants in the HIVnegative arm, the direction and magnitude of the relationships between senescent CD4 cells with cfPWV and crAiX were different in the two groups. This suggests that our findings may be specific to lean PLWH as opposed to lean persons in general. This may explain the observed inverse relationship of CD8+ T-cell activation and CD4+ T-cell senescence and arterial stiffness in PLWH with a low BMI.

The augmentation index is said to be a composite measure of wave reflection and arterial stiffness, and has been suggested to be a more sensitive marker for endothelial function compared to $\mathrm{PWV}^{27}$ The median values of cfAiX and crAiX were more than twice higher in PLWH than the HIV-negative persons. The significant difference observed in the cfAiX by HIV status may indicate that endothelial dysfunction is more pronounced in more elastic vessels as compared to more muscular vessels. Carotid femoral pulse wave velocity is a measure of arterial stiffness in elastic vessels, whereas crPWV in muscular vessels. ${ }^{26} \mathrm{~A}$ decrease in vascular compliance has been linked to reduced bioavailability of $\mathrm{NO}$, which has several physiological roles, including vasodilation of coronary arteries..$^{33}$ Nitric oxide diffuses through the vascular smooth muscle cells where it activates guanylate cyclase, a process that leads to smooth muscle relaxation. The viral protein Nef has been implicated in the reduction of endothelial NO, an increase in the secretion of cytokines from macrophages and inducing endothelial cell apoptosis. ${ }^{34}$ Using a rat model, Kline, Kleinhenz ${ }^{18}$ reported an association between the HIV proteins and a decrease in vascular and systemic NO bioavailability. This study also reported endothelial dysfunction because of the inability to relax maximally after the injection of acetylcholine. The decrease in NO was reported not to be from eNOS regulation but from the overproduction of superoxide binding to increased NO 


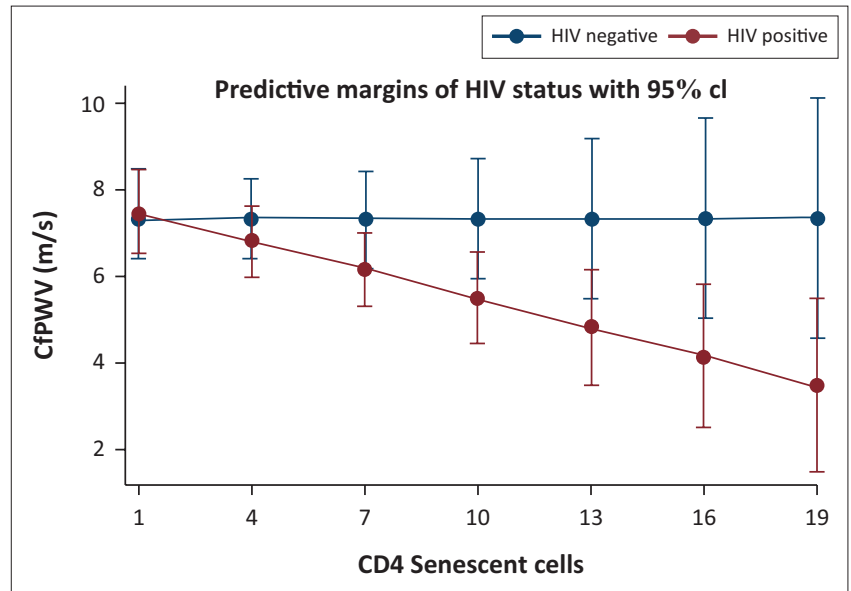

cfPWV, carotid-femoral pulse wave velocity; HIV, human immunodeficiency virus; $\mathrm{Cl}_{\text {, }}$ confidence interval; CD4, cluster of differentiation 4.

FIGURE 1: Relationship of carotid-femoral pulse wave velocity and senescent CD4 $T$ cells by human immunodeficiency virus status. Senescent CD4+ cells are shown as the proportion of total CD4+ cells. The model was adjusted for age and body mass index.

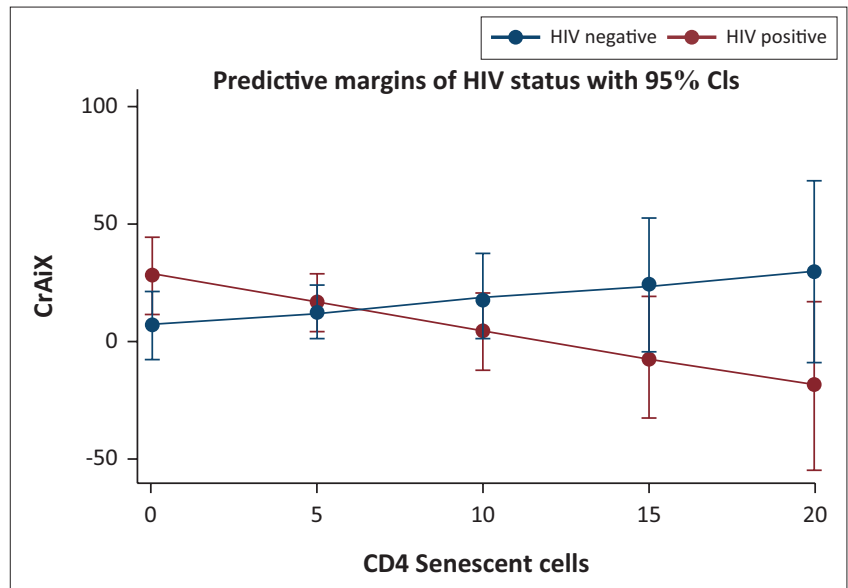

crAiX, carotid-radial augmentation index; $\mathrm{HIV}$, human immunodeficiency virus; $\mathrm{Cl}$, confidence interval; CD4, cluster of differentiation 4.

FIGURE 2: Relationship of carotid-radial augmentation index and senescent CD4 $T$ cells by human immunodeficiency virus status. Senescent CD4+ cells are shown as the proportion of total CD4+ cells. The model was adjusted for age and body mass index.

to form peroxynitrite. However, even though increased in PLWH, our study found no significant difference in 8-isoprostane levels in PLWH.

We observed an inverse association between CD4-activated T cells and 8-isoprostane, suggesting immune activation may not be a driver of increased oxidative stress in lean PLWH. However, the directionality of this relationship could not be assessed in our study. This is in contrast with other studies that reported an increase in immune activation and oxidative stress markers in PLWH as compared to HIV-negative persons. ${ }^{35}$ Mandas, Iorio $^{36}$ reported both an increase in oxidative stress markers and a decrease in antioxidants in the PLWH when compared to the HIV-negative persons.

The cohort of PLWH had been on anti-retroviral therapy for more than 5 years and was assumed to be virally suppressed. 
However, we observed persistent immune activation of both CD4+ and CD8+ T cells in the PLWH.

With CD4+ and CD8+ T cells being the main components of the immune response, their activation has been associated with disease progression, particularly in those who are treatment naïve. ${ }^{37,38,39}$ Circulating activated immune cells can permeate through to the adventitia and adhere to the endothelium resulting in its dysfunction. ${ }^{13}$ The median values of both activation markers on CD4+ and CD8+ T cells were more than double in PLWH when compared to the HIV-negative persons. We found a significant association between crPWV and activated CD8+ T cells. Paradoxically, this association was inversely related in PLWH and directly related in HIV-negative persons. This draws our attention to the nature of the vasculature being investigated and whether the immune response to elastic versus muscular vessels is different.

The study also saw an inverse association between CD4 senescence markers and cfPWV. Persons living with HIV on treatment or naïve have been described to be immunologically aged when compared to HIV-negative persons, and this has been associated with increased immune activation. ${ }^{40,41}$ This has been attributed to a decrease in the telomere length in DNA molecules. ${ }^{40,41}$ Telomere shortening leads to the initiation of the DNA damage response leading to growth arrest, which if not repaired, leads to permanent growth arrest that is irreversible. At this stage, the cells are neither dead nor functional.

Prior studies of vascular compliance and immune function have mainly been conducted in individuals with higher BMI in cohorts outside of SSA.9,12,13 Visceral fat, being metabolically active, is an important site for the secretion of adipokines that influence inflammation, lipid metabolism and insulin sensitivity. ${ }^{42,43}$

Lukich, Gavish ${ }^{42}$ suggested measuring abdominal fat content by waist circumference, as it is a more sensitive measure of endothelial dysfunction than overall body mass measured by BMI. Although our study population was all lean, PLWH had a significantly lower BMI compared to HIV-negative persons. However, waist circumferences were similar between the two groups, potentially reflecting greater visceral obesity in the participants with HIV. ${ }^{44}$

\section{Strengths and limitations}

Our study groups had a far lower median BMI $\left(18.9 \mathrm{~kg} / \mathrm{m}^{2}\right.$ in the PWLH and $20.7 \mathrm{~kg} / \mathrm{m}^{2}$ in the HIV-negatives) as compared to similar studies from cohorts in the US and other settings, and we excluded smokers and persons with known cardiovascular pathology. We also assessed a range of T-cell phenotypes and multiple indices of vascular compliance. However, a major limitation of our study is the significantly greater age and small sample size amongst the PLWH compared to HIV-negatives in our study, which precludes attributing the lack of a similar association between T-cell activation and vascular compliance to HIV status. Also, information on the duration of HIV infection before treatment and total CD4 and CD8 T-cell counts were not available.
Furthermore, because of the cross-sectional design, causality could not be determined and may not be generalisable as a result of scarcity of information linking these parameters in the African context. Additional longitudinal studies will be needed to explore mechanisms linking immune activation and endothelial dysfunction. ${ }^{45}$

\section{Conclusion}

Lean adults with HIV had increased immune activation and arterial stiffness when compared to HIV-negative persons. Paradoxically, lower arterial stiffness was associated with increasing CD8+ T-cell activation and CD4+ T-cell senescence, in contrast to prior studies in predominantly US cohorts with high proportions of overweight and obese participants. To date, there have been few studies of immune activation and arterial stiffness amongst PLWH in SSA, and further research is needed to determine whether these findings are specific to PLWH with a low BMI or to individuals in SSA in general. Furthermore, the implications of these findings in the context of rising rates of CVD amongst PLWH in the region should be explored further.

\section{Acknowledgements}

The authors would like to specially thank the staff at TROPGAN laboratory and the NUSTART centre at the University Teaching Hospital, Lusaka.

\section{Competing interests}

The authors declare that they have no financial or personal relationships that may have inappropriately influenced them in writing this article.

\section{Authors' contributions}

L.K., F.G., D.C.H. and J.R.K. conceived and planned the experiments. L.K. and T.C. carried out the experiments. L.K., T.C. and S.M. contributed to sample preparation. L.K., F.G., C.G., S.M., W.M., D.C.H. and J.R.K. contributed to the interpretation of the results. L.K. took the lead in writing the manuscript. All authors provided critical feedback and helped shape the research, analysis and manuscript.

\section{Funding information}

This work was supported by the Fogarty International Centre of the U.S. National Institutes of Health under the Award Number D43 TW009744, the NIH-funded Vanderbilt Clinical and Translational Science Award from NCRR/NIH Grant UL1 RR024975, the NIH-funded Tennessee Centre for AIDS Research Grant P30 AI110527, and National Institutes of Health grant K01HL130497. The content is solely the responsibility of the authors and does not necessarily represent the official views of the National Institutes of Health.

\section{Data availability}

The data that support the findings of this study are available from the corresponding author, L.K., upon reasonable request. 


\section{Disclaimer}

The views and opinions expressed in this article are those of the authors and do not necessarily reflect the official policy or position of any affiliated agency of the authors.

\section{References}

1. Yaya S, Ekholuenetale M, Bishwajit G. Differentials in prevalence and correlates of metabolic risk factors of non-communicable diseases among women in subSaharan Africa: Evidence from 33 countries. BMC Public Health. 2018;18(1):1168 https://doi.org/10.1186/s12889-018-6085-2

2. Yuyun MF, Sliwa K, Kengne AP, Mocumbi AO, Bukhman G. Cardiovascular disease in sub-Saharan Africa compared to high-income countries: An epidemiological perspective. Glob Heart. 2020;15(1):15. https://doi.org/10.5334/gh.403

3. Fourie CMT, Botha-Le Roux S, Smith W, et al. Vascular function and cardiovascular risk in a HIV infected and HIV free cohort of African ancestry: Baseline profile, rationale and methods of the longitudinal EndoAfrica-NWU study. BMC Infect Dis. 2020;20(1):473. https://doi.org/10.1186/s12879-020-05173-6

4. Losina E, Hyle EP, Borre ED, et al. Projecting 10-year, 20-year, and lifetime risks of cardiovascular disease in persons living with human immunodeficiency virus in the United States. Clin Infect Dis. 2017;65(8):1266-1271. https://doi.org/10.1093/ cid/cix547

5. Cerrato E, D'Ascenzo F, Biondi-Zoccai G, et al. Cardiac dysfunction in pauci symptomatic human immunodeficiency virus patients: A meta-analysis in the highly active antiretroviral therapy era. Eur Heart J. 2013;34(19):1432-1436. https://doi.org/10.1093/eurheartj/ehs471

6. Masenga SK, Hamooya BM, Nzala S, et al. Patho-immune mechanisms of hypertension in HIV: A systematic and thematic review. Curr Hypertens Rep. 2019;21(7):56. https://doi.org/10.1007/s11906-019-0956-5

7. Vlachopoulos C, Aznaouridis K, Stefanadis C. Prediction of cardiovascular events and all-cause mortality with arterial stiffness: A systematic review and metaanalysis. J Am Coll Cardiol. 2010;55(13):1318-1327. https://doi.org/10.1016/j. jacc.2009.10.061

8. Kaplan RC, Sinclair E, Landay AL, et al. T cell activation and senescence predict subclinical carotid artery disease in HIV-infected women. J Infect Dis. 2011;203(4):452-463. https://doi.org/10.1093/infdis/jiq071

9. Karim R, Mack WJ, Kono N, et al. T-cell activation, both pre- and post-HAART levels, correlates with carotid artery stiffness over 6.5 years among HIV-infected women in the WIHS. J Acquir Immune Defic Syndr. 2014;67(3):349-356. https:// doi.org/10.1097/QAl.0000000000000311

10. Evans TG, Rasmussen K, Wiebke G, Hibbs Jr, JB. Nitric oxide synthesis in patients with advanced HIV infection. Clin Exp Immunol. 1994;97(1):83-86. https://doi. org/10.1111/j.1365-2249.1994.tb06583.x

11. Koethe JR, Heimburger DC, PrayGod G, Filteau S. From wasting to obesity: The contribution of nutritional status to immune activation in HIV infection. J Infect contribution of nutritional status to immune activation in HIV infection.
Dis. 2016;214 Suppl 2:S75-S82. https://doi.org/10.1093/infdis/jiw286

12. Kaplan RC, Sinclair E, Landay AL, et al. T cell activation predicts carotid artery stiffness among HIV-infected women. Atherosclerosis. 2011;217(1):207-213. https://doi.org/10.1016/j.atherosclerosis.2011.03.011

13. Grome HN, Barnett L, Hagar CC, Harrison DG, Kalams SA, Koethe JR. Association of T cell and macrophage activation with arterial vascular health in HIV. AIDS Res Hum Retroviruses. 2017;33(2):181-186. https://doi.org/10.1089/aid.2016.0113

14. Longenecker $\mathrm{CT}$, Funderburg NT, Jiang $\mathrm{Y}$, et al. Markers of inflammation and CD8 T-cell activation, but not monocyte activation, are associated with subclinical carotid artery disease in HIV-infected individuals. HIV Med. 2013;14(6):385-390. https://doi.org/10.1111/hiv.12013

15. Porter KM, Sutliff RL. HIV-1, reactive oxygen species, and vascular complications Free Radic Biol Med. 2012;53(1):143-159. https://doi.org/10.1016/j. freeradbiomed.2012.03.019

16. Tejero J, Shiva S, Gladwin MT. Sources of vascular nitric oxide and reactive oxygen species and their regulation. Physiol Rev. 2019;99(1):311-379. https://doi. org/10.1152/physrev.00036.2017

17. Ganong W. Review of medical physiology. 22nd ed. s.l.: McGraw Hill Companies; 2005.

18. Kline ER, Kleinhenz DJ, Liang B, et al. Vascular oxidative stress and nitric oxide depletion in HIV-1 transgenic rats are reversed by glutathione restoration. Am J Physiol Heart Circ Physiol. 2008;294(6):H2792-H2804. https://doi.org/10.1152/ ajpheart.91447.2007

19. Musisi E, Matovu DK, Bukenya A, et al. Effect of anti-retroviral therapy on oxidative stress in hospitalized HIV-infected adults with and without TB. Afr Health Sci. 2018;18(3):512-522. https://doi.org/10.4314/ahs.v18i3.7

20. Panee J, Pang X, Munsaka S, Berry MJ, Chang L. Independent and co-morbid HIV infection and meth use disorders on oxidative stress markers in the cerebrospinal fluid and depressive symptoms. J Neuroimmune Pharmacol. 2015;10(1):111-121. https://doi.org/10.1007/s11481-014-9581-x
21. Masia M, Padilla S, Fernandez M, et al. Oxidative stress predicts all-cause mortality in HIV-infected patients. PLOS One. 2016:11(4):e0153456. https://doi. org/10.1371/journal.pone.0153456

22. Low H, Hoang A, Pushkarsky $T$, et al. HIV disease, metabolic dysfunction and atherosclerosis: A three year prospective study. PLoS One. 2019;14(4):e0215620. https://doi.org/10.1371/journal.pone.0215620

23. Shah ASV, Stelzle D, Lee KK, et al. Global burden of atherosclerotic cardiovascular disease in people living with HIV: Systematic review and meta-analysis. Circulation. 2018;138(11):1100-1112. https://doi.org/10.1161/CIRCULATIONAHA.117.033369

24. Jaacks LM, Vandevijvere S, Pan A, et al. The obesity transition: Stages of the global epidemic. Lancet Diabetes Endocrinol. 2019;7(3):231-240. https://doi. org/10.1016/S2213-8587(19)30026-9

25. WHO. WHO STEPwise approach to surveillance, WHO STEPS instrument. 2003. Available from: https://www.who.int/ncds/surveillance/steps/instrument/en/

26. Mitchell GF, Hwang SJ, Vasan RS, et al. Arterial stiffness and cardiovascular events: The Framingham Heart Study. Circulation. 2010;121(4):505-511. https://doi. org/10.1161/CIRCULATIONAHA.109.886655

27. Afsar B, Elsurer R. The relationship between magnesium and ambulatory blood pressure, augmentation index, pulse wave velocity, total peripheral resistance, and cardiac output in essential hypertensive patients. J Am Soc Hypertens. 2014;8(1):28-35. https://doi.org/10.1016/j.jash.2013.10.006

28. Alam M. Complior analyse; Measure of pulse wave velocity and central pressure analysis operator manual. Alam medical v1.9beta version; 2013.

29. Biosciences B. Direct immunofluoresence staining of whole blood using a lyse/ wash procedure [homepage on the Internet]. 2002 [cited 2020 Oct. 15]. Available from: https://www.bdbiosciences.com/us/resources/s/stainlysewash

30. R\&D D. 8-isoprostane oxidative stress ELISA kit [homepage on the Internet]. 1997 [cited 2020 Oct. 15]. Available from: https://www.detroitrandd.com/sites/ default/files/documents/8_iso_spec_sheet_120219.pdf

31. Schutte AE, Huisman HW, Schutte R, et al. Arterial stiffness profiles: Investigating various sections of the arterial tree of African and Caucasian people. Clin Exp Hypertens. 2011;33(8):511-517. https://doi.org/10.3109/10641963.2011.561897

32. Ngatchou $W$, Lemogoum $D$, Ndobo $P$, et al. Increased burden and severity of metabolic syndrome and arterial stiffness in treatment-naive HIV+ patients from Cameroon. Vasc Health Risk Manag. 2013;9:509-516. https://doi.org/10.2147/ VHRM.S42350

33. Caballero AE. Endothelial dysfunction in obesity and insulin resistance: A road to diabetes and heart disease. Obes Res. 2003;11(11):1278-1289. https://doi. org/10.1038/oby.2003.174

34. Nou E, Lo J, Grinspoon SK. Inflammation, immune activation, and cardiovascular disease in HIV. AIDS. 2016;30(10):1495-1509. https://doi.org/10.1097/ QAD.0000000000001109

35. Chettimada S, Lorenz DR, Misra V, et al. Exosome markers associated with immune activation and oxidative stress in HIV patients on antiretroviral therapy. Sci Rep. 2018;8(1):7227. https://doi.org/10.1038/s41598-018-25515-4

36. Mandas A, lorio EL, Congiu MG, et al. Oxidative imbalance in HIV-1 infected patients treated with antiretroviral therapy. J Biomed Biotechnol. 2009;2009:749575. https://doi.org/10.1155/2009/749575

37. Paiardini M, Muller-Trutwin M. HIV-associated chronic immune activation. Immunol Rev. 2013;254(1):78-101. https://doi.org/10.1111/imr.12079

38. Deeks SG, Kitchen CM, Liu L, et al. Immune activation set point during early HIV infection predicts subsequent CD4+ T-cell changes independent of viral load. Blood. 2004;104(4):942-947. https://doi.org/10.1182/blood-2003-09-3333

39. Hsu DC, Sereti I. Serious non-AIDS events: Therapeutic targets of immune activation and chronic inflammation in HIV infection. Drugs. 2016;76(5):533-549. https://doi.org/10.1007/s40265-016-0546-7

40. Warren JA, Clutton G, Goonetilleke N. Harnessing CD8(+) T cells under HIV antiretroviral therapy. Front Immunol. 2019;10:291. https://doi.org/10.3389/ fimmu.2019.00291

41. Gianesin K, Noguera-Julian A, Zanchetta $M$, et al. Premature aging and immune senescence in HIV-infected children. AIDS. 2016;30(9):1363-1373. https://doi. org/10.1097/QAD.0000000000001093

42. Lukich A, Gavish D, Shargorodsky M. Normal weight diabetic patients vs obese diabetics relation of overall and abdominal adiposity to vascular health. Cardiovasc Diabetol. 2014;13:141. https://doi.org/10.1186/s12933-014-0141-8

43. Gustafson B, Hammarstedt A, Andersson CX, Smith U. Inflamed adipose tissue: A culprit underlying the metabolic syndrome and atherosclerosis. Arterioscle Thromb Vasc Biol. 2007;27(11):2276-2283. https://doi.org/10.1161/ ATVBAHA.107.147835

44. Shoelson SE, Lee J, Goldfine AB. Inflammation and insulin resistance. J Clin Invest. 2006;116(7):1793-1801. https://doi.org/10.1172/JCl29069

45. Spyridopoulos I, Martin-Ruiz C, Hilkens C, et al. CMV seropositivity and T-cell senescence predict increased cardiovascular mortality in octogenarians: Results from the Newcastle 85+ study. Aging Cell. 2016;15(2):389-392. https://doi. org/10.1111/acel.12430 

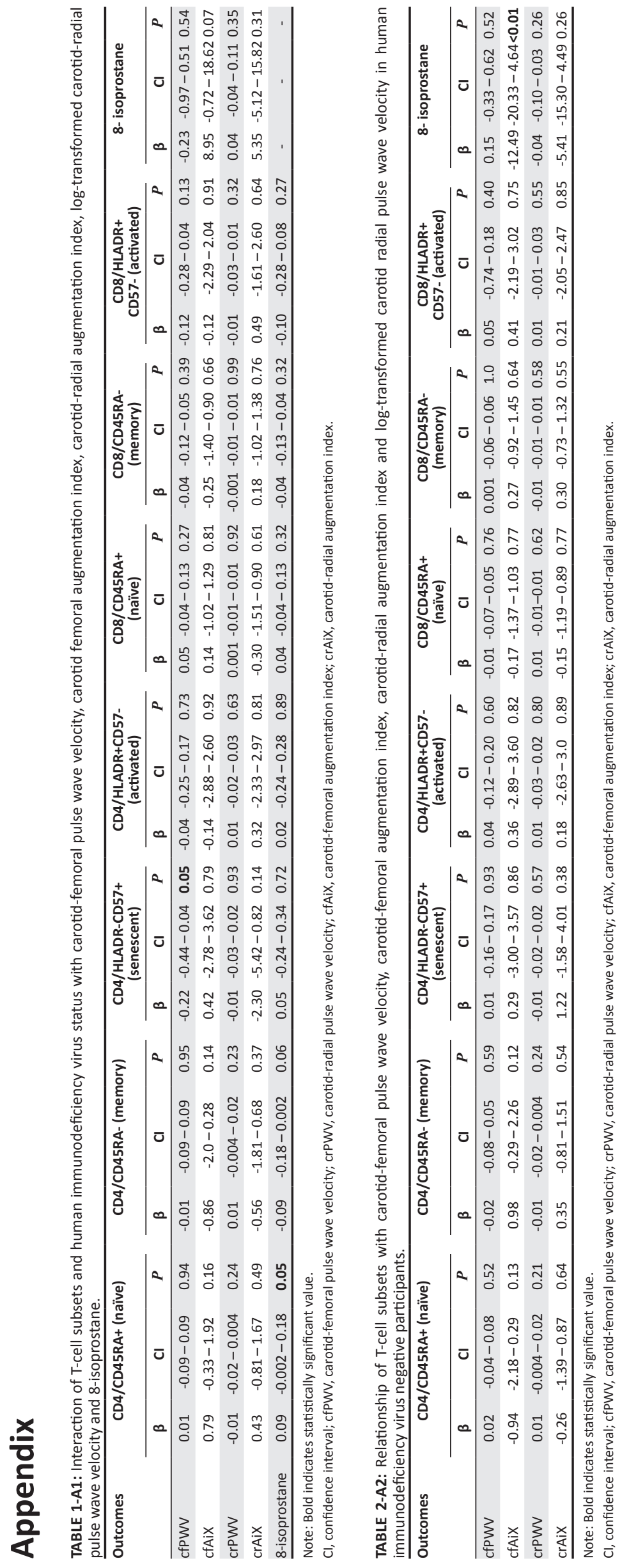
TABLE 3-A3: Analysis of variance table for the interaction effect of CD4 senescent cells and human immunodeficiency virus on carotid-femoral pulse wave velocity.

\begin{tabular}{lccc}
\hline Variable & Degrees of freedom & $\boldsymbol{F}$ & $\boldsymbol{P}$ \\
\hline Age & 1 & 1.66 & 0.21 \\
BMI & 1 & 0.99 & 0.33 \\
HIV status & 1 & 0.20 & 0.66 \\
CD4 senescent cells & 1 & 0.00 & 0.99 \\
HIV status: CD4 senescent cells & 1 & 4.21 & 0.05 \\
interaction & & & \\
Regression & 5 & 2.65 & 0.04 \\
\hline Total & $\mathbf{5 1}$ & - & - \\
\hline
\end{tabular}

BMI, body mass index; HIV, human immunodeficiency virus; CD4, cluster of differentiation 4.
TABLE 4-A4: Analysis of variance table for the interaction effect of senescent CD4 $T$ cells and human immunodeficiency virus on carotid-radial augmentation index.

\begin{tabular}{lccc}
\hline Variable & Degrees of freedom & $\boldsymbol{F}$ & $\boldsymbol{P}$ \\
\hline Age & 1 & 3.22 & 0.08 \\
BMI & 1 & 1.93 & 0.17 \\
HIV status & 1 & 3.23 & 0.08 \\
CD4 senescent cells & 1 & 1.03 & 0.32 \\
HIV status: CD4 senescent cells interaction & 1 & 4.81 & 0.03 \\
Regression & 5 & 3.23 & 0.01 \\
\hline Total & $\mathbf{5 1}$ & - & -
\end{tabular}

BMI, body mass index; HIV, human immunodeficiency virus; $C D 4$, cluster of differentiation 4 .

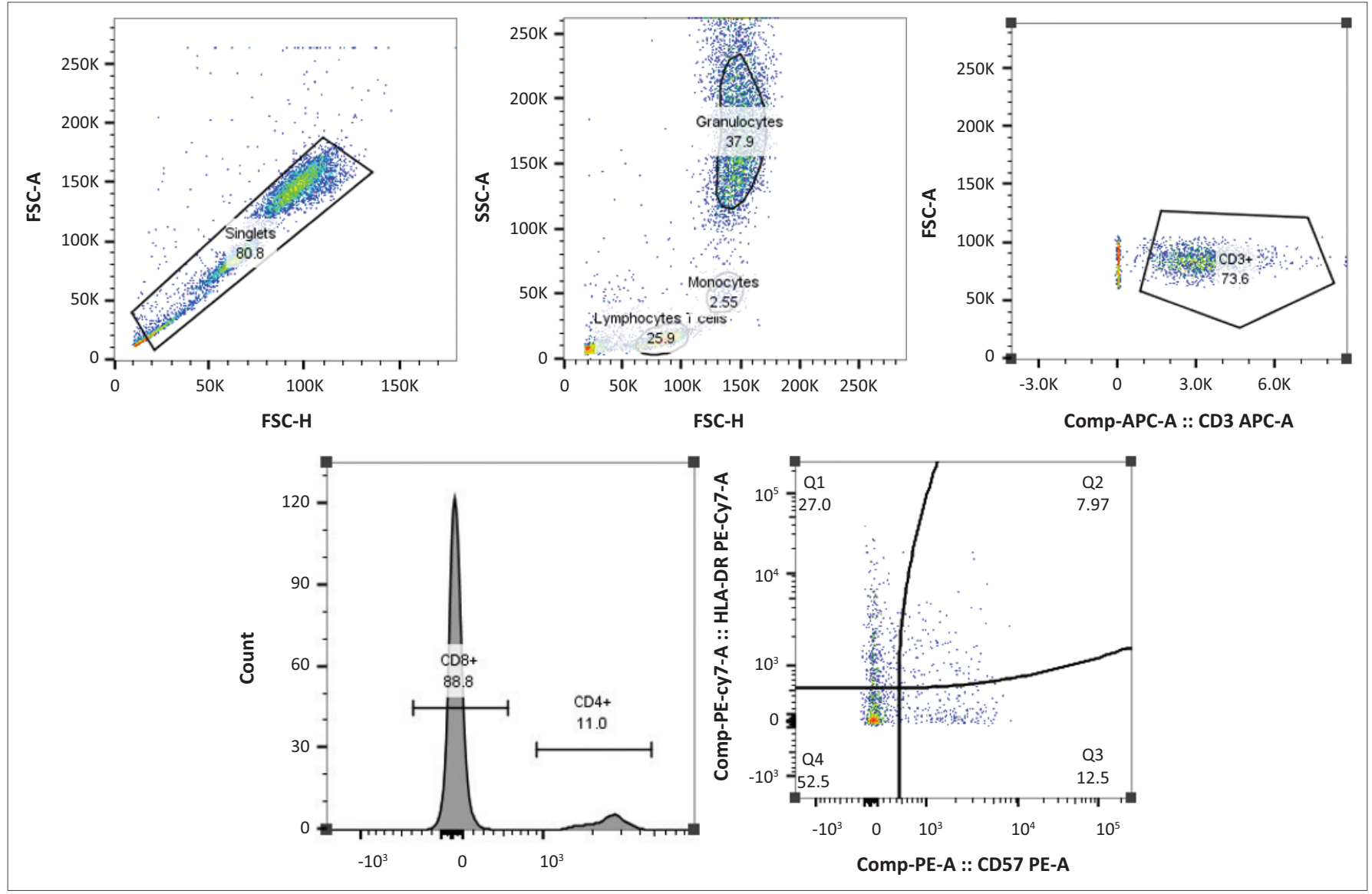

FIGURE 1-A1: T-cell gating strategy. 